\title{
Self-affine Graphene Metasurfaces for Tunable Broadband Absorption
}

\author{
Pin Chieh $\mathrm{Wu}^{1,2}$, Nikitas Papasimakis ${ }^{3, *}$, and Din Ping Tsai ${ }^{1,2, *}$ \\ ${ }^{1}$ Department of Physics, National Taiwan University, Taipei 10617, Taiwan \\ ${ }^{2}$ Research Center for Applied Sciences, Academia Sinica, Taipei 11529, Taiwan \\ ${ }^{3}$ Optoelectronics Research Centre and Centre for Photonic Metamaterials, University of Southampton, \\ Southampton SO17 1BJ, UK \\ *E-mail: dptsai@sinica.edu.tw, n.papasimakis@soton.ac.uk
}

\begin{abstract}
Graphene has emerged as a promising platform for $\mathrm{THz}$ metasurfaces supporting electrically tunable deep-subwavelength plasmonic excitations. Here, we introduce a broadband graphene metasurface based on the Hilbert curve, a continuous, space-filling fractal. We demonstrate enhancement of graphene absorption over a broad frequency band (0.5-60 THz) with an average absorption level exceeding 20\%. Owing to the continuous nature of the metasurface patterns, both absorption level and bandwidth can be controlled electrically by varying the graphene charge carrier concentration.
\end{abstract}

Keywords: graphene plasmonics, fractal metasurfaces, broadband absorber 
Structuring materials [1-4] at the subwavelength scale has led to the emergence of metamaterials [5-7], a paradigm shift in the design of artificial electromagnetic materials [8] resulting in technologically important, as well as exotic, applications, including perfect lenses [9], transformation optics and invisibility cloaks [10], and perfect absorbers [11]. The properties of metamaterials largely arise from resonant dispersion; hence material loss restricts the strength [12] and bandwidth of the metamaterial response [13]. In particular, with respect to perfect absorbers, various schemes have been suggested in order to achieve strong absorption in gigahertz [14], terahertz [15] and visible [16] parts of the spectrum. In most cases, a metasurface (a single metamaterial layer [17]) is combined with a metallic mirror separated by a dielectric spacer of subwavelength thickness [18-20]. The bandwidth of such an absorber is generally narrow due to its resonance nature. Typical approaches include combining multiple narrow absorption lines by vertically stacking metasurfaces [21-23], incorporating resonators with different resonance frequencies into the metasurface unit cell [24], or simply employing multiple plasmonic resonances of a single resonator [25]. However, such methods often result in complex, bulky configurations, with little tunability. Graphene, a monolayer of carbon atoms, provides an appealing alternative: owing to its band structure, graphene supports plasmonic excitations at the deep sub-wavelength scale [26]. Plasmonic resonators can be realized by structuring graphene at the nanoscale [27-29], which can then be employed to construct graphene metasurfaces targeting the $\mathrm{THz}$ part of the spectrum [30-34]. At the same time the high sensitivity of the electromagnetic properties of graphene to the charge carrier [35] concentration provides means of electrical control of plasmons [36-38]. In 
addition to tunability, graphene as a plasmonic material allows to strongly confine electromagnetic radiation to much smaller physical volumes compared to typical metals. For example, the plasmon wavelength for graphene is about 50 times smaller than the free-space wavelength, while for noble metals this is an order of magnitude smaller [26, 29]. Such strong field confinement allows people to realize very complex graphene structures while retaining a subwavelength footprint. Graphene exhibits broadband nonlinearities and high thermal conductivity, whereas its atomic thickness facilitates device integration.

Here, we introduce fractal graphene metasurfaces as tunable, broadband $\mathrm{THz}$ absorbers of monoatomic thickness (see Fig. 1(a)). We demonstrate multifold enhancement of absorption over an extremely broad frequency band, where average absorption levels exceed $20 \%$ from 0.5 to $60 \mathrm{THz}$ in strongly doped graphene. We show that the absorption band is strongly dependent on the charge carrier concentration enabling dynamic electrostatic control of the metasurface properties.

The Hilbert curve, a continuous fractal space-filling curve [39], can be constructed by following simple iterative rules (see Fig. 1(b)): starting with a U shaped element, at each level the curve is composed from the previous level by a combined scaling, translation and rotation operation, resulting in patterns of increasing density and complexity. In the limit where the process is repeated to infinity, the Hilbert curve fully fills the 2D plane, while remaining essentially one-dimensional. Owing to its hierarchical structuring, the Hilbert curve has been employed in the design of broadband electromagnetic components 
allowing for long electrical lengths and small footprints. Examples include multiband antenna arrays [40], high impedance metasurfaces [41], isotropic broadband metamaterials [42], transparent electrodes [43], and substrates for surface enhanced Raman scattering [44]. Moreover, metallic metasurfaces with Hilbert curve and other fractal designs have been combined with graphene, in order to realize absorbers [45] and photodetectors [46]. Here, we demonstrate wideband operation of a single-layer graphene absorber with a Hilbert design. At all levels of the Hilbert curve studied here, the footprint remains fixed at $3.2 \times 3.2 \mu \mathrm{m}^{2}$. The linewidth is considered to be $15 \mathrm{~nm}$, which is accessible with standard fabrication methods [29].

A representative image of strong absorption in a fractal single-layer graphene metasurface in this work is shown in Fig. 1(a). The fractal graphene metasurface is illuminated by an electromagnetic wave with frequency within the resonance band of the structure. As a result of resonant plasmon excitation and accompanying enhanced local electromagnetic fields (hot spots), the incident wave is strongly absorbed. We study numerically metamaterial arrays of such elements, where each unit cell comprises a single fractal curve. We consider two complementary families of graphene Hilbert structures (positive and negative) of levels I to V (see Fig. 1(b)) and study numerically their absorption behavior under normal incidence illumination. All calculations are performed by a finite element solver (COMSOL). Graphene was modeled as an infinitely thin layer with surface conductivity according to the Drude model. By neglecting 
interband transitions as well as the effect of temperature $(\mathrm{T}=0)$ the conductivity of graphene is given by $[27,30,47]$

$$
\sigma=\frac{e^{2} E_{f}}{\pi^{2}} \frac{i}{\omega+i \gamma}
$$

Where $e$ is the electron charge, $E_{f}$ is the Fermi energy and $\gamma=e v_{F}^{2} / \mu E_{f}$ is the damping rate. Here, $v_{F}=10^{6} \mathrm{~m} / \mathrm{s}$ is the Fermi velocity [48] and $\mu$ is the mobility. In our simulations, the mobility varies between 500 and $10,000 \mathrm{~cm}^{2} / \mathrm{Vs}$, typical values for largescale graphene fabricated by chemical vapour deposition [49]. By substituting for the doping level (Fermi energy) and frequency in Eq. (1), the complex, frequency dependent permittivity is readily obtained. The range of values for key parameters, such as the Drude weight and damping rate are listed in the Supporting Information (Table S1). These values are consistent with experimental data $[47,50]$.

The absorption spectra for positive and negative structures are shown in Figs. 2(a) and 2(b), respectively, for levels I, III and V at moderate graphene doping levels (corresponding to a Fermi energy of $0.5 \mathrm{eV}$ or a carrier concentration of $\sim 1.83 \times 10^{13} \mathrm{~cm}^{-}$ ${ }^{2}$ with a mobility of $10,000 \mathrm{~cm}^{2} / \mathrm{Vs}$ ). In essence, level I is a split-ring resonator, a common building block for metamaterials and metasurfaces, exhibiting a well-defined resonance at $2.27 \mathrm{THz}$, which is related to the total length of the graphene wire. Less pronounced higher-order resonances are visible at 3.74 and $5.02 \mathrm{THz}$ (see Fig. 2(a), top panel). The complementary (negative) structure (see Fig. 2(b)) under illumination with the orthogonal polarization (electric field along the y-axis) exhibits a similar series of resonances, with much higher absorption levels reaching $\sim 50 \%$, the limit for planar structures [28]. By increasing the level of the Hilbert curve, the absorption spectrum 
becomes more complex with multiple resonances covering an increasingly broad frequency range for both positive and negative designs. At the same time the fundamental resonance moves to increasingly lower frequencies due to the longer electrical length of the metasurface. Illumination of the positive (negative) Hilbert metasurfaces with the orthogonal polarization, along the y-axis, (x-axis) results in a similar behavior (see Supporting Information).

To investigate the origin of the resonant behavior of the graphene Hilbert curves, we present in Fig. 3 the electric and magnetic field distributions in the vicinity of the graphene layer (for the three lowest-order resonances) for both positive and negative graphene Hilbert structures of levels I, III and V. We illustrate the resonance excitations of the positive Hilbert curves by looking at the z-component of the electric near-field distribution, which indicates the areas of charge concentration. Similarly, in the case of negative Hilbert curves, the excitations can be understood by looking at (fictional) magnetic charges $[51,52]$ as illustrated by the z-component of the magnetic field. The first level of the positive Hilbert curve is similar to a split-ring resonator (SRR) and as such it shows a fundamental resonance, with a half-wavelength standing wave current configuration $\left(\lambda_{p} / 2\right.$, where $\lambda_{p}$ is the effective graphene plasmon wavelength) along its length (see Fig. 3(a) top row, left panel). Its complementary structure, a SRR-shaped aperture demonstrates a similar behavior, with effective 'magnetic currents' replacing the electric ones (see Fig. 3(b) top row, left panel). At higher-order resonances, the electric (magnetic) field maps for the positive (negative) Hilbert curves of level I indicate an increasing number of nodes of the standing wave excitation (see Figs. 3(a) \& 3(b), left 
columns). In the case of higher levels (see Figs. 3(a) and 3(b), middle and right columns), the length of the Hilbert curve increases considerably and the excitations rapidly become complex, with different regions of the structure resonating at different frequencies.

The broadband response of the graphene Hilbert curve depends strongly on both the intrinsic loss (mobility) and doping levels. In Fig. 4(a), we show the dependence of the absorption spectrum of the graphene Hilbert metasurface (level V) under y-polarized illumination on the mobility, assuming a $0.5 \mathrm{eV}$ fixed doping. At low mobility values, the absorption resonances are broad and partially overlap. Increasing the mobility leads to decrease of dissipation loss and hence narrower resonances. At the same time, however, the graphene metasurface scatters more strongly leading to substantial radiation loss because of the stronger reflection associated with higher mobility (see Supporting Information). The dependence of the absorption spectrum on doping is shown in Figs. 4(b) \& 4(c). In contrast to the absorption spectra of unstructured graphene which depend weakly on the doping levels (filled circles, Fig. 4(c)), in the case of the Hilbert metasurface increasing the number of charge carriers in graphene affects both the strength and position of the resonances. As the Fermi energy is increased, resonances blue-shift, as expected from Drude model considerations [27]. This resonance shift can also be realized by the change of carrier density. As graphene layer doped with more carrier densities, resonance features with electron-hole pair transition occur under photon absorption with higher energy, resulting in a resonance blue-shift. This attributes to the intrinsic property of graphene and therefore independent with graphene structures [47, 53]. The absorption enhancement can be explained by considering that in the absence of 
quenching, increasing the number of carriers effectively increases the probability of virtual electron-hole pair transition by photon absorption.

The performance of the graphene Hilbert absorbers can be quantified by the absorption bandwidth (defined as the frequency range over which the absorption of the metasurface is higher than the absorption of unstructured graphene) and by the average absorption over this bandwidth. The dependence of these metrics on the level of the Hilbert curve is shown in Fig. 5. Increasing the level of the Hilbert curve leads to substantial enhancement of the absorption bandwidth, as a result of the multi-resonant spectral features (see Fig. 5(a)). At the same time, the average absorption displays a nonmonotonic behavior with a peak of $\sim 22.5 \%$ for the level II negative curve. This is attributed to the increase in length of the pattern with increasing the level of the Hilbert curve, which renders the fundamental and lower-order resonances inaccessible as they redshift outside the frequency range of our calculations. On the other hand, increasing the levels of doping leads to a substantial enhancement of the average absorption from $\sim 3 \%$ at $0.05 \mathrm{eV}$ to $\sim 22 \%$ at $1 \mathrm{eV}$ over a broad bandwidth of $\sim 60 \mathrm{THz}$ (see Fig. 5(b)). This is a direct result of the blueshift of the metasurface's absorption resonances with increasing doping, hence allowing to excite lower-order modes.

The graphene metasurfaces studied in this work exhibit remarkably high values of absorption, when one considers their monoatomic thickness and that, in contrast to typical approaches [28], no metallic mirror is present. In the case of a negative level I 
Hilbert metasurface (essentially an array of complementary SRRs) resonant absorption reaches $50 \%$, which is the limit for planar structures in a symmetric environment. The negative level V metasurface exhibits an absorption bandwidth as wide as $60 \mathrm{THz}$ (almost 190\% of the center frequency) covering the mid-infrared and terahertz spectral range with a $\sim 22 \%$ average absorption. To the best of our knowledge, this is the highest absorption value over such a broad bandwidth in an atomically thin layer [5, 28, 54-57]. Varying the graphene Fermi energy level within a routinely accessible range $(<1 \mathrm{eV})$ leads to order of magnitude changes in both absorption levels and bandwidth. Further improvements in the absorption levels can be achieved by coupling the graphene Hilbert metasurface to other types of broadband resonant systems [58, 59], although leading to less compact and more complex configurations. Finally, our results offer a way towards the development of tunable $\mathrm{THz}$ absorbers enabling applications in sensing and energy harvesting.

In summary, we have investigated the $\mathrm{THz}$ response of both positive and negative graphene Hilbert metasurfaces of levels from I to $\mathrm{V}$ throughout the mid-infrared and terahertz range. We show numerically that graphene fractal metasurfaces can exhibit a broad absorption band that can be dynamically controlled by adjusting the Fermi energy level. Because of its atomic thickness, current fabrication techniques are capable of achieving the $15 \mathrm{~nm}$ linewidth assumed in our numerical studies. The fabrication imperfections results in two main effects: small shift of the resonance frequencies and inhomogeneous broadening, and increase losses by increasing scattering. The former is of little consequence for our aims, where we target broadband operation, while the second is 
expected to be negligible, due to the large mismatch between the plasmonic and the freespace wavelengths (two orders of magnitude difference). It is worth emphasizing that owing to the tunability of graphene, our approach can be combined with dynamic modulation of conductivity, enabling applications in polarization modulation [60] with high operation speed. The time-dependent conductivity is also promising for the development of tunable frequency comb generation [61]. These results offer a way towards the development of tunable absorbers enabling applications in terahertz and infrared detection and modulation for graphene photonics and optoelectronics.

\section{Acknowledgments}

The authors acknowledge financial support from Ministry of Science and Technology, Taiwan (Grant No. MOST-104-2745-M-002-003-ASP) and Academia Sinica (Grant No. AS-103-TP-A06). They are also grateful to National Center for Theoretical Sciences, Molecular Imaging Center of National Taiwan University, National Center for HighPerformance Computing, Taiwan, and Research Center for Applied Sciences, Academia Sinica, Taiwan for their supports. The authors also acknowledge the support of the UK's

Engineering and Physical Sciences Research Council (grant EP/M008797/1) and the Leverhulme Trust. 
(a)

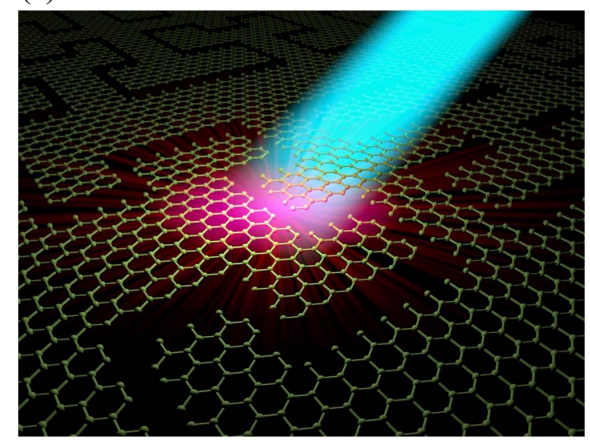

(b)

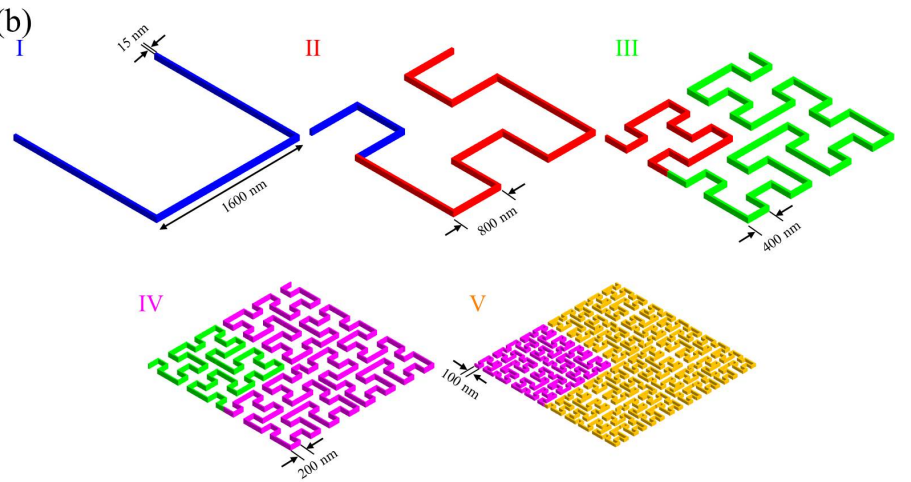

FIG. 1. (a) Schematic illustration of strongly absorbing Hilbert graphene metasurfaces. (b) Iterative construction of the Hilbert curve, for the first five (I-V) levels. Each level is constructed from the previous one by scaling, translation and rotation as indicated by the color scheme. For all levels, the curve is contained in a $3.2 \times 3.2 \mu \mathrm{m}^{2}$ area, while its linewidth is $15 \mathrm{~nm}$. 
(a)

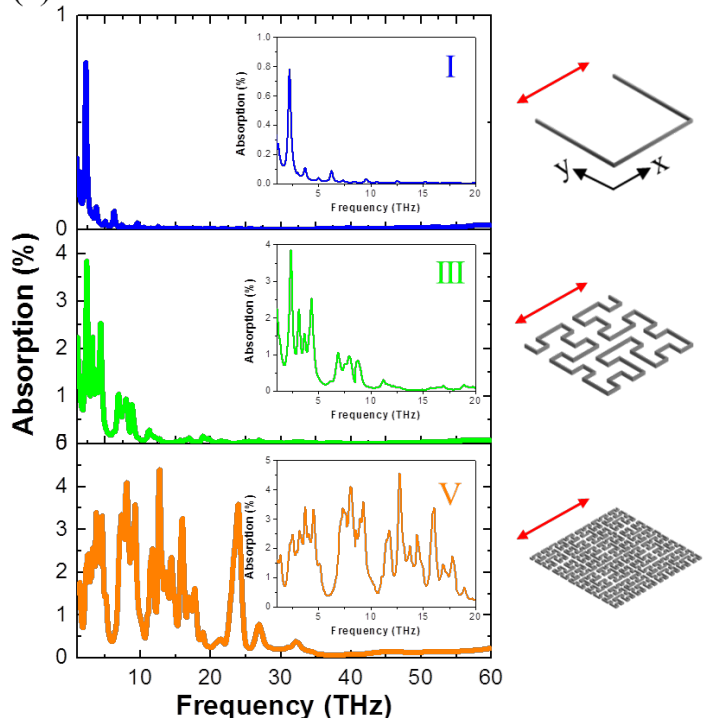

(b)

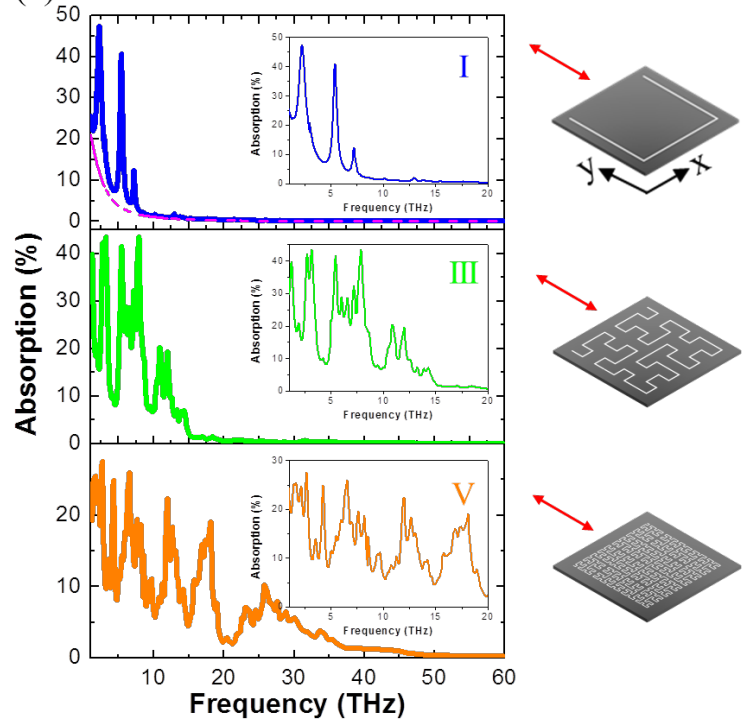

FIG. 2. Simulated absorption spectra for (a) positive and (b) negative graphene Hilbert structures of levels I, III and V. Insets show the low frequency region of the absorption spectra. Schematics on the right side show the corresponding graphene structures, where red arrows indicate the electric field orientation of the incident wave. The magenta dashed line in (b, top panel) represents the absorption of unstructured graphene for comparison. The Fermi energy is $0.5 \mathrm{eV}$ for all presented cases, which corresponds to a charge carrier concentration of $\sim 1.83 \times 10^{13}\left(\mathrm{~cm}^{-2}\right)$. Absorption spectra of all five levels can be found in the Supporting Information. 


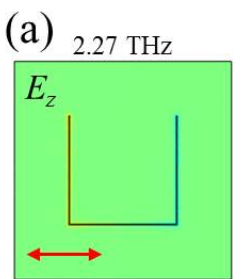

$3.74 \mathrm{THz}$

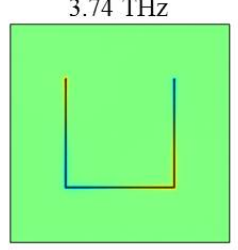

$5.02 \mathrm{THz}$

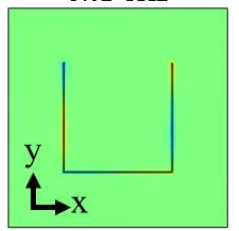

$1.07 \mathrm{THz}$

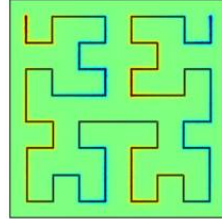

$2.41 \mathrm{THz}$

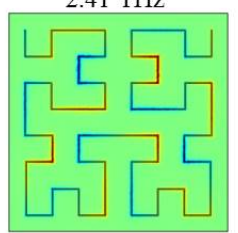

$3.18 \mathrm{THz}$

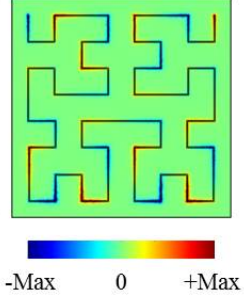

(b)

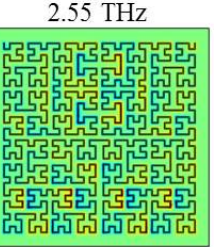

$3.21 \mathrm{THz}$

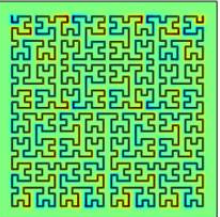

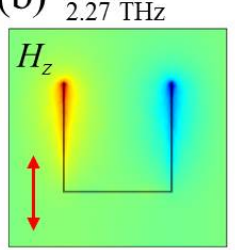

$5.43 \mathrm{THz}$

$1.27 \mathrm{THz}$
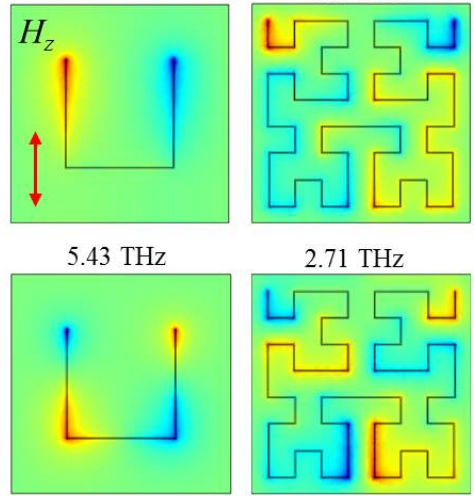

$7.17 \mathrm{THz}$

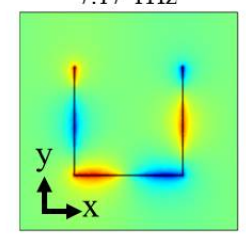

$2.71 \mathrm{THz}$

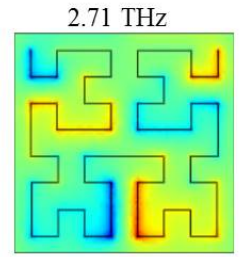

$3.17 \mathrm{THz}$

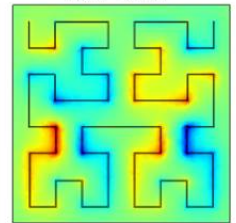

$1.51 \mathrm{THz}$

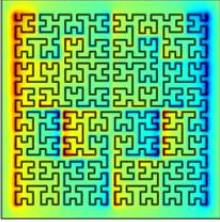

$2.15 \mathrm{THz}$

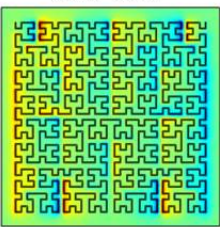

$2.70 \mathrm{THz}$

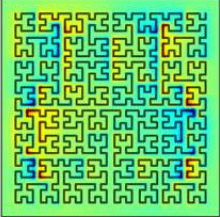

-Max $0 \quad+$ Max

FIG. 3. The electromagnetic field maps for (a) positive and (b) graphene Hilbert structures (of level I, III and $\mathrm{V}$ ) at three lowest order resonances (from top to bottom). In order to clearly identify the resonant modes, $E_{z}$ and $H_{z}$ are shown for positive and negative graphene Hilbert structures, respectively. Red arrows: direction of incident electric field. 

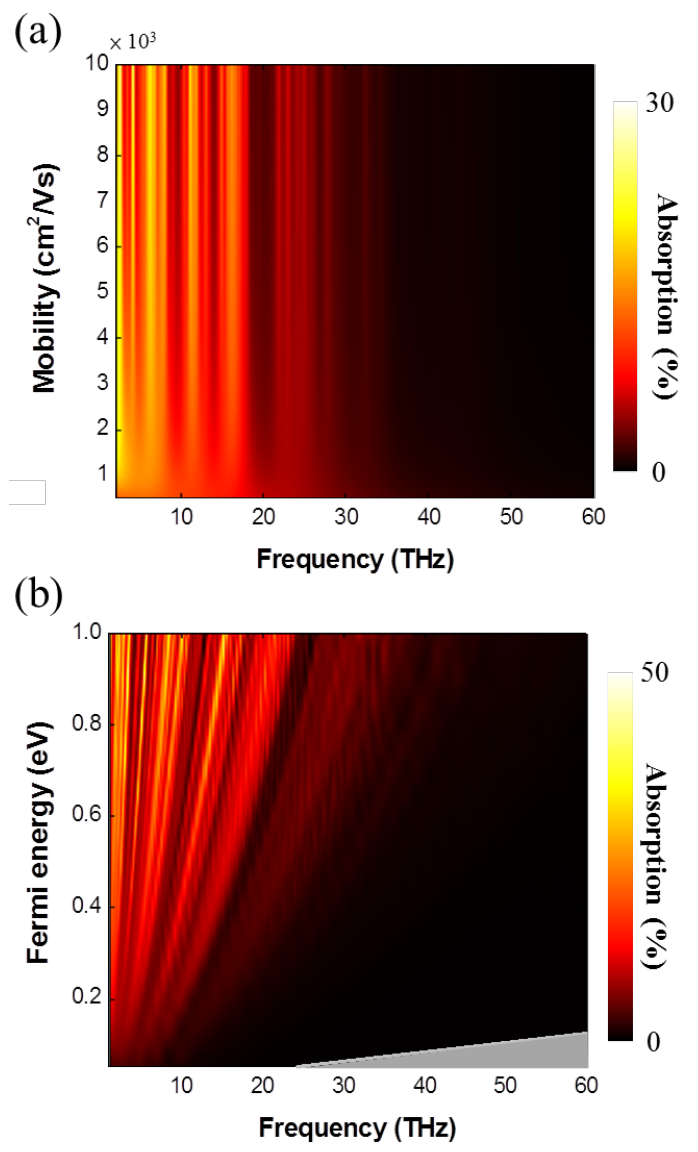

(c)

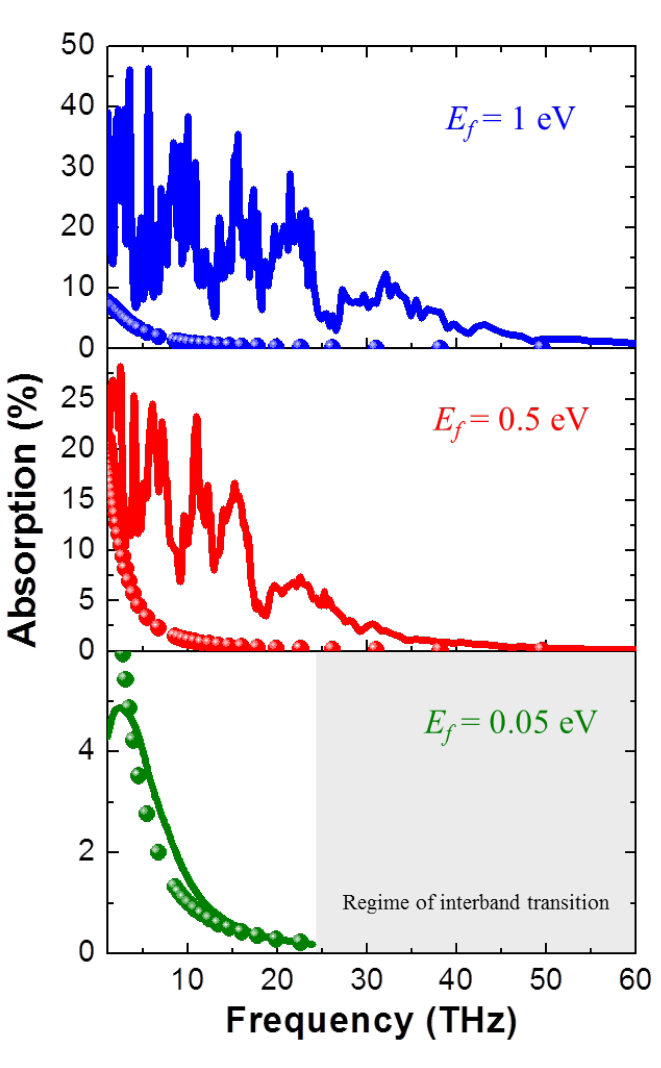

FIG. 4. Absorption of the level V negative graphene Hilbert structures under y-polarized illumination with varying (a) mobility and (b) Fermi energy. The Fermi energy and mobility is fixed at $0.5 \mathrm{eV}$ and 10,000 $\mathrm{cm}^{2} / \mathrm{Vs}$ for (a) and (b), respectively. The grey areas indicate the non-validity region of the Drude model due to the onset of interband transition. (c) Cross-sections from (b) for Fermi energies $0.05 \mathrm{eV}$ (olive), $0.5 \mathrm{eV}$ (red) and $1 \mathrm{eV}$ (blue). Solid lines: negative graphene Hilbert structures. Dots: unstructured graphene layer. 
(a)

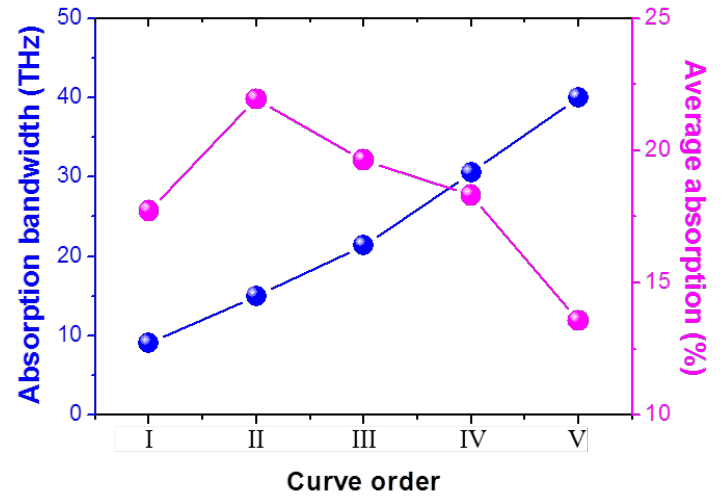

(b)

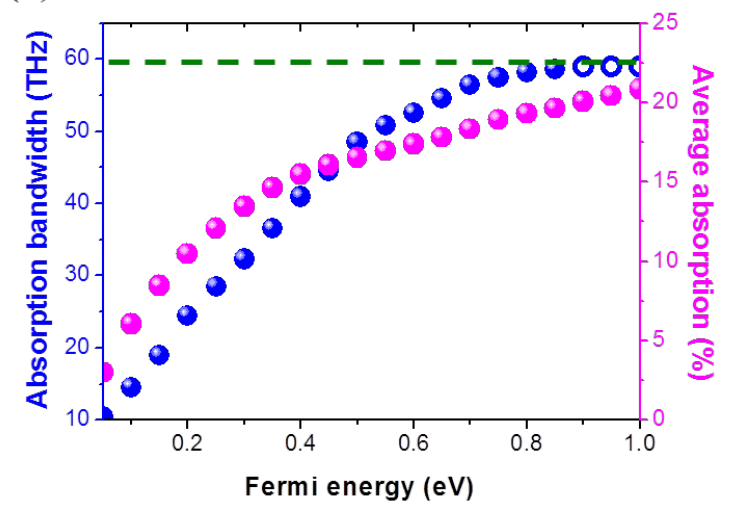

FIG. 5. Absorption bandwidth (blue curves) and average absorption (magenta curves) as a function of (a) the Hilbert curve level and (b) Fermi energy. The level V negative graphene Hilbert structure is considered for (b). The olive dash line indicates the upper limit of the frequency range used in our simulations. All cases are studied by using y-polarized illumination. In both (a) and (b) the mobility is fixed at 10,000 $\mathrm{cm}^{2} /$ Vs. In (a) the Fermi energy is fixed at $0.5 \mathrm{eV}$. 


\section{References}

[1] H.-P. Wang, D.-H. Lien, M.-L. Tsai, C.-A. Lin, H.-C. Chang, K.-Y. Lai, and J.-H. He, Photon management in nNanostructured solar cells, J. Mater. Chem. C 2, 3144-3171 (2014).

[2] W. C. Luk, K. M. Yeung, K. C. Tam, K. L. Ng, K. C. Kwok, C. Y. Kwong, A. M. C. Ng, and A. B. Djurišić, Enhanced conversion efficiency of polymeric photovoltaic cell by nanostructured antireflection coating, Org. Electron. 12, 557-561 (2011).

[3] W. T. Chen, P. C. Wu, C. J. Chen, C.-J. Weng, H.-C. Lee, T.-J. Yen, C.-H. Kuan, M. Mansuripur, and D. P. Tsai, Manipulation of multidimensional plasmonic spectra for information storage, Appl. Phys. Lett. 98, 171106-171103 (2011).

[4] C. C. Chen, C. T. Hsiao, S. Sun, K.-Y. Yang, P. C. Wu, W. T. Chen, Y. H. Tang, Y.-F. Chau, E. Plum, G.-Y. Guo, N. I. Zheludev, and D. P. Tsai, Fabrication of three dimensional split ring resonators by stressdriven assembly method, Opt. Express 20, 9415-9420 (2012).

[5] N. I. Zheludev, and Y. S. Kivshar, From metamaterials to metadevices, Nat. Mater. 11, 917-924 (2012).

[6] P. C. Wu, G. Sun, W. T. Chen, K.-Y. Yang, Y.-W. Huang, Y.-H. Chen, H. L. Huang, W.-L. Hsu, H. P. Chiang, and D. P. Tsai, Vertical split-ring resonator based nanoplasmonic sensor, Appl. Phys. Lett. 105, 033105 (2014).

[7] N. Papasimakis, V. A. Fedotov, V. Savinov, T. A. Raybould, and N. I. Zheludev, Electromagnetic toroidal excitations in matter and free space, Nat. Mater. 15, 263-271 (2016).

[8] V. V. Klimov, and D. V. Guzatov, Plasmonic atoms and plasmonic molecules, Appl. Phys. A 89, 305314 (2007).

[9] J. B. Pendry, Negative refraction makes a perfect lens, Phys. Rev. Lett. 85, 3966-3969 (2000).

[10] J. B. Pendry, D. Schurig, and D. R. Smith, Controlling electromagnetic fields, Science 312, 1780-1782 (2006).

[11] C. M. Watts, X. Liu, and W. J. Padilla, Metamaterial electromagnetic wave absorbers, Adv. Mater. 24, OP98-OP120 (2012).

[12] Z.-G. Dong, H. Liu, J.-X. Cao, T. Li, S.-M. Wang, S.-N. Zhu, and X. Zhang, Enhanced sensing performance by the plasmonic analog of electromagnetically induced transparency in active metamaterials, Appl. Phys. Lett. 97, 114101 (2010).

[13] G. V. Naik, V. M. Shalaev, and A. Boltasseva, Alternative plasmonic materials: Beyond gold and silver, Adv. Mater. 25, 3264-3294 (2013).

[14] N. I. Landy, S. Sajuyigbe, J. J. Mock, D. R. Smith, and W. J. Padilla, Perfect metamaterial absorber, Phys. Rev. Lett. 100, 207402 (2008). 
[15] W. Withayachumnankul, C. M. Shah, C. Fumeaux, B. S. Y. Ung, W. J. Padilla, M. Bhaskaran, D. Abbott, and S. Sriram, Plasmonic resonance toward terahertz perfect absorbers, ACS Photonics 1, 625-630 (2014).

[16] K. Aydin, V. E. Ferry, R. M. Briggs, and H. A. Atwater, Broadband polarization-independent resonant light absorption using ultrathin plasmonic super absorbers, Nat. Commun. 2, 517 (2011).

[17] Y.-W. Huang, W. T. Chen, W.-Y. Tsai, P. C. Wu, C.-M. Wang, G. Sun, and D. P. Tsai, Aluminum plasmonic multicolor meta-Hologram, Nano Lett. 15, 3122-3127 (2015).

[18] X. Liu, T. Starr, A. F. Starr, and W. J. Padilla, Infrared spatial and frequency selective metamaterial with near-unity absorbance, Phys. Rev. Lett. 104, 207403 (2010).

[19] N. Liu, M. Mesch, T. Weiss, M. Hentschel, and H. Giessen, Infrared perfect absorber and its application as plasmonic sensor, Nano Lett. 10, 2342-2348 (2010).

[20] A. Tittl, P. Mai, R. Taubert, D. Dregely, N. Liu, and H. Giessen, Palladium-based plasmonic perfect absorber in the visible wavelength range and its application to hydrogen sensing, Nano Lett. 11, 4366-4369 (2011).

[21] Y. Cui, K. H. Fung, J. Xu, H. Ma, Y. Jin, S. He, and N. X. Fang, Ultrabroadband light absorption by a sawtooth anisotropic metamaterial slab, Nano Lett. 12, 1443-1447 (2012).

[22] M. L. Tseng, P. C. Wu, S. Sun, C. M. Chang, W. T. Chen, C. H. Chu, P. L. Chen, L. Zhou, D. W. Huang, T. J. Yen, and D. P. Tsai, Fabrication of multilayer metamaterials by femtosecond laser-induced forward-transfer technique, Laser Photon. Rev. 6, $702-707$ (2012).

[23] N. R. Han, Z. C. Chen, C. S. Lim, B. Ng, and M. H. Hong, Broadband multi-layer terahertz metamaterials fabrication and characterization on flexible substrates, Opt. Express 19, 6990-6998 (2011).

[24] J. Zhu, Z. Ma, W. Sun, F. Ding, Q. He, L. Zhou, and Y. Ma, Ultra-broadband terahertz metamaterial absorber, Appl. Phys. Lett. 105, 021102 (2014).

[25] J. Wang, C. Fan, P. Ding, J. He, Y. Cheng, W. Hu, G. Cai, E. Liang, and Q. Xue, Tunable broad-band perfect absorber by exciting of multiple plasmon resonances at optical frequency, Opt. Express 20, 1487114878 (2012).

[26] M. Jablan, H. Buljan, and M. Soljačić, Plasmonics in graphene at infrared frequencies, Phys. Rev. B 80, 245435 (2009).

[27] F. J. García de Abajo, Graphene plasmonics: Challenges and opportunities, ACS Photonics 1, 135-152 (2014).

[28] S. Thongrattanasiri, F. H. L. Koppens, and F. J. García de Abajo, Complete optical absorption in periodically patterned graphene, Phys. Rev. Lett. 108, 047401 (2012). 
[29] V. W. Brar, M. S. Jang, M. Sherrott, J. J. Lopez, and H. A. Atwater, Highly confined tunable midinfrared plasmonics in graphene nanoresonators, Nano Lett. 13, 2541-2547 (2013).

[30] N. Papasimakis, S. Thongrattanasiri, N. I. Zheludev, and F. J. Garcia de Abajo, The magnetic response of graphene split-ring metamaterials, Light Sci Appl 2, e78 (2013).

[31] Y. Fan, Z. Liu, F. Zhang, Q. Zhao, Z. Wei, Q. Fu, J. Li, C. Gu, and H. Li, Tunable mid-infrared coherent perfect absorption in a graphene meta-surface, Sci. Rep. 5, 13956 (2015).

[32] Y. Fan, N.-H. Shen, T. Koschny, and C. M. Soukoulis, Tunable terahertz meta-surface with graphene cut-wires, ACS Photonics 2, 151-156 (2015).

[33] Q. Zhang, Q. Ma, S. Yan, F. Wu, X. He, and J. Jiang, Tunable terahertz absorption in graphene-based metamaterial, Opt. Commun. 353, 70-75 (2015).

[34] R. Ning, J. Bao, Z. Jiao, and Y. Xu, Omnidirectional polarization-insensitive tunable absorption in graphene metamaterial of nanodisk structure, J. Appl. Phys. 118, 203101 (2015).

[35] K. S. Novoselov, A. K. Geim, S. V. Morozov, D. Jiang, Y. Zhang, S. V. Dubonos, I. V. Grigorieva, and A. A. Firsov, Electric field effect in atomically thin carbon films, Science 306, 666-669 (2004).

[36] Z. Fei, A. S. Rodin, G. O. Andreev, W. Bao, A. S. McLeod, M. Wagner, L. M. Zhang, Z. Zhao, M. Thiemens, G. Dominguez, M. M. Fogler, A. H. C. Neto, C. N. Lau, F. Keilmann, and D. N. Basov, Gatetuning of graphene plasmons revealed by infrared nano-imaging, Nature 487, 82-85 (2012).

[37] J. Chen, M. Badioli, P. Alonso-Gonzalez, S. Thongrattanasiri, F. Huth, J. Osmond, M. Spasenovic, A. Centeno, A. Pesquera, P. Godignon, A. Zurutuza Elorza, N. Camara, F. J. G. de Abajo, R. Hillenbrand, and F. H. L. Koppens, Optical nano-imaging of gate-tunable graphene plasmons, Nature 481, 77-81 (2012).

[38] A. Ishikawa, and T. Tanaka, Plasmon hybridization in graphene metamaterials, Appl. Phys. Lett. 102, 253110-253114 (2013).

[39] Hilbert, D. Über die stetige Abbildung einer Linie auf ein Flächenstück, Mathematische Annalen, 38, 459-460 (1989).

[40] D. H. Werner, and P. L. Werner, Frequency-independent features of self-similar fractal antennas, Radio Sci. 31, 1331-1343 (1996).

[41] A. Karmakar, S. Verma, M. Pal, and R. Ghatak, An Ultra-wideband Monopole Antenna with Multiple Fractal Slots with Dual Band Rejection Characteristics, Progress In Electromagnetics Research C 31, 185197 (2012).

[42] S. De Zuani, T. Reindl, M. Rommel, B. Gompf, A. Berrier, and M. Dressel, High-order hilbert curves: fractal structures with isotropic, tailorable optical properties, ACS Photonics 2, 1719-1724 (2015).

[43] F. Afshinmanesh, A. G. Curto, K. M. Milaninia, N. F. van Hulst, and M. L. Brongersma, Transparent metallic fractal electrodes for semiconductor devices, Nano Lett. 14, 5068-5074 (2014). 
[44] I. Grigorenko, Nanostructures with the Hilbert curve geometry as surface enhanced Raman scattering substrates, Appl. Phys. Lett. 103, 043123 (2013).

[45] X. Huang, Z. Hu, and P. Liu, Graphene based tunable fractal Hilbert curve array broadband radar absorbing screen for radar cross section reduction, AIP Advances 4, 117103 (2014).

[46] S. Gottheim, H. Zhang, A. O. Govorov, and N. J. Halas, Fractal nanoparticle plasmonics: The cayley tree, ACS Nano 9, 3284-3292 (2015).

[47] Z. Fang, S. Thongrattanasiri, A. Schlather, Z. Liu, L. Ma, Y. Wang, P. M. Ajayan, P. Nordlander, N. J. Halas, and F. J. García de Abajo, Gated tunability and hybridization of localized plasmons in nanostructured graphene, ACS Nano 7, 2388-2395 (2013).

[48] A. Manjavacas, P. Nordlander, and F. J. García de Abajo, Plasmon blockade in nanostructured graphene, ACS Nano 6, 1724-1731 (2012).

[49] X. Li, W. Cai, J. An, S. Kim, J. Nah, D. Yang, R. Piner, A. Velamakanni, I. Jung, E. Tutuc, S. K. Banerjee, L. Colombo, and R. S. Ruoff, Large-area synthesis of high-quality and uniform graphene films on copper foils, Science 324, 1312-1314 (2009).

[50] P. Tassin, T. Koschny, and C. M. Soukoulis, Graphene for terahertz applications, Science 341, 620621 (2013).

[51] F. Falcone, T. Lopetegi, M. A. G. Laso, J. D. Baena, J. Bonache, M. Beruete, R. Marques, F. Martin, and M. Sorolla, Babinet principle applied to the design of metasurfaces and metamaterials, Phys. Rev. Lett. 93 (2004).

[52] A. Bitzer, A. Ortner, H. Merbold, T. Feurer, and M. Walther, Terahertz near-field microscopy of complementary planar metamaterials: Babinet's principle, Opt. Express 19, 2537-2545 (2011).

[53] B. Vasic, G. Isic, and R. Gajic, Localized surface plasmon resonances in graphene ribbon arrays for sensing of dielectric environment at infrared frequencies, J. Appl. Phys. 113, 013110-013117 (2013).

[54] J. R. Piper, and S. Fan, Total Absorption in a graphene monolayer in the optical regime by critical coupling with a photonic crystal guided resonance, ACS Photonics 1, 347-353 (2014).

[55] Z. Fang, Y. Wang, A. E. Schlather, Z. Liu, P. M. Ajayan, F. J. García de Abajo, P. Nordlander, X. Zhu, and N. J. Halas, Active tunable 002.absorption enhancement with graphene nanodisk arrays, Nano Lett. 14, 299-304 (2013).

[56] R. Alaee, M. Farhat, C. Rockstuhl, and F. Lederer, A perfect absorber made of a graphene microribbon metamaterial, Opt. Express 20, 28017-28024 (2012).

[57] Y. Fan, Z. Wei, Z. Zhang, and H. Li, Enhancing infrared extinction and absorption in a monolayer graphene sheet by harvesting the electric dipolar mode of split ring resonators, Opt. Lett. 38, 5410-5413 (2013). 
[58] K. Bhattarai, Z. Ku, S. Silva, J. Jeon, J. O. Kim, S. J. Lee, A. Urbas, and J. Zhou, A large-area, mushroom-capped plasmonic perfect absorber: Refractive index sensing and Fabry-Perot cavity mechanism, Adv. Opt. Mater. 3, 1779-1786 (2015).

[59] H.-T. Chen, Interference theory of metamaterial perfect absorbers, Opt. Express 20, 7165-7172 (2012).

[60] Z. Miao, Q. Wu, X. Li, Q. He, K. Ding, Z. An, Y. Zhang, and L. Zhou, Widely tunable terahertz Phase modulation with gate-controlled graphene metasurfaces, Phys. Rev. X 5, 041027 (2015).

[61] V. Ginis, P. Tassin, T. Koschny, and C. M. Soukoulis, Tunable terahertz frequency comb generation using time-dependent graphene sheets, Phys. Rev. B 91, 161403 (2015). 